Supporting Information

\title{
Synthesis of Nanowires via Temperature-Induced Supramolecular Step-Growth Polymerization
}

Hongbing Gao,,$^{\dagger}$ Xiaodong Ma, ${ }^{\dagger}$ Jiaping Lin, * Liquan Wang, Chunhua Cai, * Liangshun Zhang, and Xiaohui Tian

Shanghai Key Laboratory of Advanced Polymeric Materials, Key Laboratory for Ultrafine Materials of Ministry of Education, School of Materials Science and Engineering, East China University of Science and Technology, Shanghai 200237, China

\footnotetext{
* Corresponding author. E-mail: jlin@ecust.edu.cn; caichunhua@ecust.edu.cn

${ }^{\dagger}$ These authors contributed equally to this work.
} 


\section{Contents}

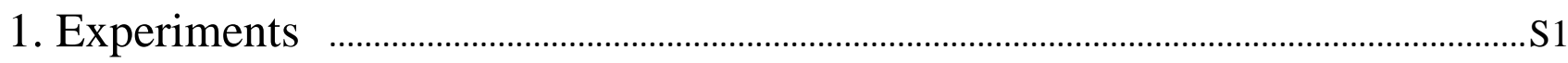

1.1 Polymer synthesis and aggregate preparation …………………………………….....

1.2. Definitions and measurements of the parameters $X_{\mathrm{n}}, f_{\mathrm{X}}, p$, and $P D I \ldots \ldots \ldots \ldots \ldots \ldots \ldots . . . . \mathrm{S} 7$

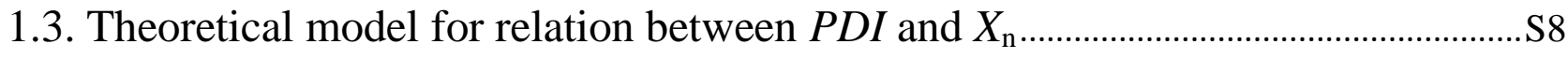

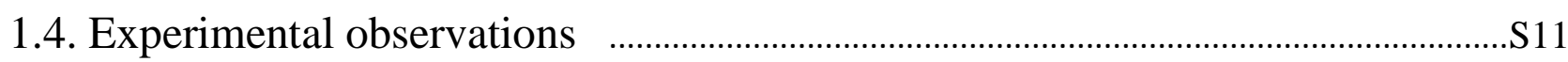

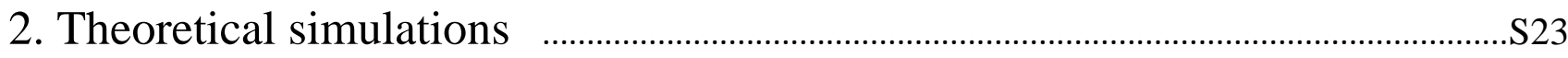

2.1 Dissipative particle dynamics (DPD) method ......................................................... 223

2.2 Simulation model and parameter settings ……………………………………….....S25 


\section{Experiments}

\subsection{Polymer synthesis and aggregate preparation}

1.1.1 Reagents and materials. Polyethylene glycol monomethyl ether (mPEG-OH) $\left(M_{\mathrm{w}}=350,750\right.$, 1000, 1100) were purchased from Sigma Inc. Analytical grade of ethyl acetate, tetrahydrofuran (THF), and 1,4-dioxane were refluxed with sodium and distilled immediately before use. All the other reagents were obtained from Adamas-beta and purified according to conventional methods or used as received. The dialysis bag (Membra-cel, 3500 molecular weight cutoff) was purchased from Serva Electrophoresis GmbH.

1.1.2 Synthesis of PBLG homopolymer. $\gamma$-benzyl-L-glutamate- $N$-carboxyanhydride (BLG-NCA) was synthesized following the procedure reported in the literatures. ${ }^{[\mathrm{S} 1]}$ PBLG homopolymer was synthesized by ring opening polymerization of BLG-NCA initiated by triethylamine with 1,4-dioxane as solvent. ${ }^{[\mathrm{S} 2-\mathrm{S} 5]}$ The reaction was performed for 3 days at $15^{\circ} \mathrm{C}$. At the end of the polymerization, the viscous reaction mixture was poured into a large volume of anhydrous methanol. The precipitated product was dried under vacuum and then purified twice by repeatedly precipitating from a chloroform solution into a large volume of anhydrous methanol. The molecular weight of the PBLG homopolymers was obtained from the gel permeation chromatography (GPC) analysis (PL-GPC 50, DMF as eluent solvent). Figure S1 shows the representative GPC curves of the polymers used in this work. From the GPC curves, we obtained the molecular weight of the polymers and the polydispersity index (i.e. $M_{\mathrm{w}} / M_{\mathrm{n}}$ ). Details are provided in Table S1. 


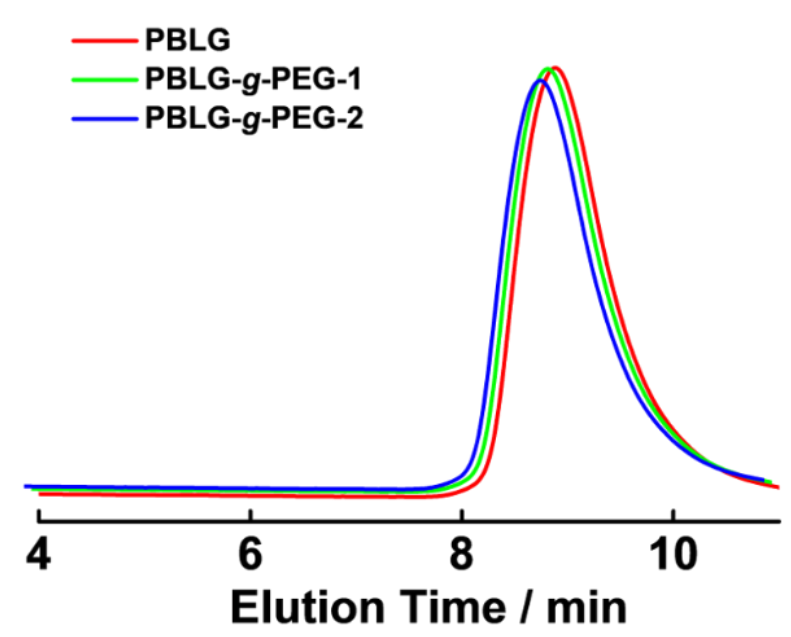

Figure S1. GPC traces of PBLG, PBLG - $g$-PEG-1 and PBLG- $g$-PEG-2 using DMF as eluent.

1.1.3 Synthesis of PBLG-g-PEG copolymers. PBLG-g-PEG graft copolymers were synthesized by ester exchange reaction of PBLG homopolymer with $\mathrm{mPEG}-\mathrm{OH} \cdot{ }^{[\mathrm{S} 3]}$ The reaction was performed at $55{ }^{\circ} \mathrm{C}$ in 1,2-dichloroethane with $p$-toluenesulfuric acid as a catalyst, and the reaction time is 1 hour. Feeding ratio of PBLG homopolymer to $\mathrm{mPEG}-\mathrm{OH}$ was 1 (i.e. $\mathrm{n}_{\mathrm{BLG}} / \mathrm{n}_{\mathrm{PEG}}, \mathrm{mol} / \mathrm{mol}, 1 / 1$ ). The reaction mixture was then precipitated in a large volume of anhydrous methanol. The product was purified twice by repeated precipitation from a chloroform solution into a large volume of anhydrous methanol and dried under vacuum.

The composition of the graft copolymer was determined by ${ }^{1} \mathrm{H}$ NMR spectrum (Avance 550, Bruker) using deuterated chloroform $\left(\mathrm{CDCl}_{3}\right)$ with 15 vol\% deuterated trifluoroacetic acid (TFA-d) as the solvent and tetramethylsilane (TMS) as the internal standard. The degree of grafting is defined as the ratio of the number of PEG chains to the degree of polymerization of the polypeptide backbone, which can be calculated from the intensity ratio of the ethylene proton signal of PEG $(\delta=$ $3.6 \mathrm{ppm})$ to that of the methylene proton signal of PBLG $(\delta=5.1 \mathrm{ppm})$ in the ${ }^{1} \mathrm{H}$ NMR spectrum. The characterization results for the structure of the PBLG-g-PEG graft copolymers were summarized in the Table S1. 
Table S1. Characteristics of the PBLG homopolymers and PBLG-g-PEG graft copolymers

\begin{tabular}{lcccccc}
\hline Sample & $\begin{array}{c}M_{\mathrm{wPBLG}}{ }^{\mathrm{a}} \\
\left(\mathrm{g} \cdot \mathrm{mol}^{-1}\right)\end{array}$ & $M_{\mathrm{w}}{ }^{\mathrm{b}}$ & $M_{\mathrm{w}} M_{\mathrm{n}}{ }^{\mathrm{c}}$ & $\begin{array}{l}M_{\mathrm{nPEG}} \\
\left(\mathrm{g} \cdot \mathrm{mol}^{-1}\right)\end{array}$ & $\begin{array}{l}\text { Degree of } \\
\text { Grafting }(\%)^{\mathrm{d}}\end{array}$ & $\begin{array}{l}\text { PEG volume } \\
\text { percentage }(\mathrm{vol} \%)\end{array}$ \\
\hline PBLG & 120000 & 120000 & 1.38 & -- & -- & - \\
PBLG-g-PEG-1 & 120000 & 120130 & 1.36 & 750 & 2.33 & 7.8 \\
PBLG-g-PEG-2 & 120000 & 120420 & 1.39 & 750 & 3.08 & 10.3 \\
PBLG-g-PEG-3 & 120000 & 120910 & 1.37 & 750 & 4.24 & 14.2 \\
PBLG-g-PEG-4 & 120000 & 121420 & 1.41 & 750 & 8.12 & 27.3 \\
PBLG-g-PEG-5 & 120000 & 120090 & 1.29 & 350 & 2.04 & 6.8 \\
PBLG-g-PEG-6 & 120000 & 120250 & 1.40 & 1000 & 2.15 & 7.2 \\
PBLG-g-PEG-7 & 120000 & 120310 & 1.34 & 1100 & 2.18 & 10.7 \\
\hline
\end{tabular}

a,b The weight average molecular weight $\left(M_{\mathrm{w}}\right)$ of PBLG homopolymer and PBLG-g-PEG graft copolymer were obtained from the gel permeation chromatography (GPC) analysis.

${ }^{\mathrm{c}}$ The polydispersity index of the polymers was determined by GPC.

${ }^{\mathrm{d}}$ The degree of grafting was calculated from the ${ }^{1} \mathrm{H}$ NMR spectra.

\subsubsection{Aggregate preparation}

The aggregates were prepared by a selective precipitation method as schematically shown in Scheme S1. First, PBLG-g-PEG graft copolymers were dissolved in THF/DMF $(1 / 1, \mathrm{v} / \mathrm{v})$ mixture solvent to obtain stock solutions. The polymer concentration of the stock solution is $0.8 \mathrm{~g} / \mathrm{L}$. Then, $1.4 \mathrm{~mL}$ of deionized water was added to $10 \mathrm{~mL}$ of the PBLG- $g$-PEG initial solution with vigorous stirring to form subunits. The experimental process to prepare subunits was kept at the constant temperature of $40{ }^{\circ} \mathrm{C}$. After stabilized for at least $10 \mathrm{~h}$, the solution was annealed respectively at 40 ${ }^{\circ} \mathrm{C}, 30{ }^{\circ} \mathrm{C}, 20{ }^{\circ} \mathrm{C}, 10{ }^{\circ} \mathrm{C}$ and $5{ }^{\circ} \mathrm{C}$. To examine the temporal variation of the micelle morphologies at each temperature, we pipetted $0.5 \mathrm{~mL}$ of sample solution into a large amount of water to freeze the morphologies every 7 days. The sample solution was dialyzed against deionized water for 3 days to 
ensure that all the organic solvents were removed. To examine the effect of the concentration of the subunits $\left(C_{\text {sub }}\right)$ on the self-assembly, we diluted the subunit solutions into various volumes using the same solvent composition (i.e., THF/DMF/water $=5 / 5 / 1.4, \mathrm{v} / \mathrm{v} / \mathrm{v}$ ). Then, TEM, AFM and Cryo-TEM were used to characterize the samples. It should be noted that after the formation of aggregates, the storage time has a negligible effect on the morphology. For example, no morphological change was observed when the micelles were aged for more than 3 months.

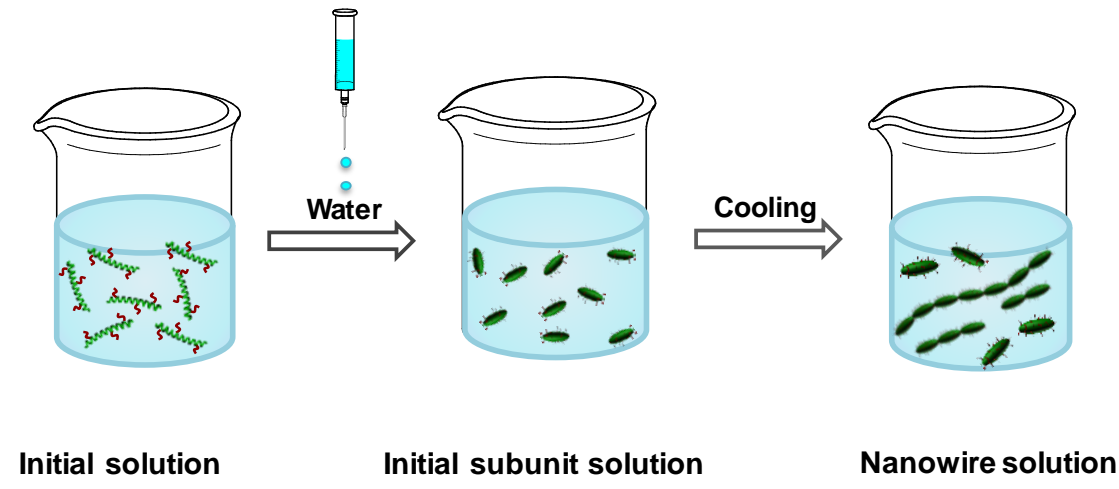

Scheme S1. Schematic representation of the aggregate preparation.

\subsubsection{Transmission Electron Microscopy (TEM)}

The morphologies of aggregates were examined by TEM (JEM-1400, JEOL) operated at an accelerating voltage of $100 \mathrm{kV}$. The sample was prepared by placing drops of solution on a copper grid coated with carbon film and then were dried at room temperature.

\subsubsection{Cryogenic Transmission Electron Microscopy (Cryo-TEM)}

Cryo-TEM samples were prepared in a controlled environment vitrification system (CEVS) at $25^{\circ} \mathrm{C}$. One drop of sample solution was placed on a copper grid coated with carbon film. The excess solution was blotted with a piece of filter paper and then quickly dipped into liquid ethane, which was cooled by liquid nitrogen. The vitrified samples were then stored in liquid nitrogen until they were transferred to a cryogenic sample holder (SN15021201W0626) and examined with a FEI-T20 
$\operatorname{TEM}(200 \mathrm{kV})$ at approximately $-174{ }^{\circ} \mathrm{C}$.

\subsubsection{Atomic Force Microscopy (AFM)}

AFM images were obtained with XE-100 AFM instrument (Park Systems), employing the tapping mode. The samples were prepared by placing one drop of the solution on a monocrystalline silicon surface, and the sample was allowed to be dried in air.

\subsubsection{Turbidity Measurements (OD)}

Turbidity measurements were carried out to determine the critical water content (CWC) for the aggregation of polymers. First, PBLG- $g$-PEG graft copolymers were dissolved in THF/DMF mixture solvent $(1 / 1$ in volume) with concentration of $0.8 \mathrm{~g} / \mathrm{L} .2 \mathrm{~mL}$ of each solution was then introduced into a quartz cell (path length: $1 \mathrm{~cm})$. Deionized water was added drop by drop $(0.01 \mathrm{~mL}$ per drop) with vigorous stirring. After each drop of deionized water was added, the solution was stirred for 1 min and then left to equilibrate for 2 min or more until the optical density was stable. The data was obtained by recording abrupt changes in turbidity during water addition. The optical density (turbidity) was measured at a wavelength of $690 \mathrm{~nm}$ with a UV-Vis spectrophotometer (UV-2550 Shimadzu).

\subsubsection{Dynamic (DLS) and Static Light Scattering (SLS) Measurements}

DLS measurements were carried out at a scattering angle of $90^{\circ}$ on a commercial LLS spectrometer (ALV/CGS-5022) equipped with an ALV-High QE APD detector and an ALV-5000 digital correlator using a He-Ne laser (the wavelength $\lambda=632.8 \mathrm{~nm}$ ) as light source. In DLS measurements, the intensity correlation function was measured at the same temperatures with that of the sample annealing. SLS measurements were performed to estimate the weight-average molecular weight of the subunits $\left(M w^{s u b}\right)$. The subunit solution for SLS measurement was prepared by dialyzing against deionized water for 3 days to ensure that all the organic solvents were removed. In 
SLS, the angular dependence of the excess absolute time-average scattered intensity of the dilute micelle aqueous solutions was measured at $25^{\circ} \mathrm{C}$. The data was collected over scattering angles of $30^{\circ}$ to $150^{\circ}$ in $2^{\circ}$ intervals. The $\mathrm{dn} / \mathrm{dc}$ value of PBLG homopolymers has been reported in the literatures. ${ }^{[\mathrm{S} 6, \mathrm{~S} 7]} \mathrm{It}$ is $0.131 \mathrm{~mL} / \mathrm{g}$ and this value is adopted in the present work. Since the mass fraction of PEG chains is low $(\sim 0.07)$ for the PBLG-g-PEG graft copolymers, the value of $0.131 \mathrm{~mL} / \mathrm{g}$ is also used for the graft copolymers. When we examined the effect of addition of PBLG homopolymer on the micellar morphologies, the $\mathrm{dn} / \mathrm{dc}$ value of $0.131 \mathrm{~mL} / \mathrm{g}$ was remained unchanged. 


\subsection{Definitions and measurements of the parameters $X_{\mathrm{n}}, f_{\mathrm{x}}, p$, and $P D I$}

\subsubsection{Number-average degree of polymerization $\left(X_{\mathrm{n}}\right)$ of the nanowires}

The parameter $X_{\mathrm{n}}$ characterizes the number-average degree of polymerization of the nanowires and is given by

$$
X_{\mathrm{n}}=\frac{\sum_{X} X n_{X}}{\sum_{X} n_{X}}
$$

where $n_{X}$ is the number of nanowires with the degree of polymerization $X$. In the experiments, the parameter $n_{X}$ was captured by mathematical statistics from the TEM images. It was obtained by collecting more than 400 aggregates and analyzing the images using Image-Pro Plus software.

\subsubsection{Number fraction $f_{X}$ of the nanowire with degree of polymerization $X$}

The parameter $f_{\mathrm{X}}$ characterizes the number fraction of the nanowires with degree of polymerization $X$ in the systems. The number fraction is given as

$$
f_{\mathrm{X}}=\frac{n_{\mathrm{X}}}{\sum_{\mathrm{X}} n_{\mathrm{X}}}
$$

\subsubsection{The extent of polymerization $(p)$}

The parameter $p$ characterizes the extent of polymerization and is given by

$$
p=\frac{\sum_{\mathrm{X}}(\mathrm{X}-1) n_{\mathrm{X}}}{\sum_{\mathrm{X}} \mathrm{X} n_{\mathrm{X}}}
$$

\subsubsection{Polydispersity index (PDI)}

The parameter $P D I$ characterizes the length distribution of the nanowires and is given by

$\mathrm{PDI}=\frac{\sum_{\mathrm{X}} \mathrm{X}^{2} n_{\mathrm{X}} \sum_{\mathrm{X}} n_{\mathrm{X}}}{\left(\sum_{\mathrm{X}} \mathrm{X} n_{\mathrm{X}}\right)^{2}}$ 


\subsection{Theoretical model for relation between $P D I$ and $X_{n}$}

In this section, we derive a detailed expression of $P D I$ by considering the activity difference of the two reactive points in a subunit. ${ }^{[88-S 11]} \mathrm{A}$ number of theoretical attempts have been given in the molecular step-growth polymerizations concerning the activity difference. Importantly, Macosko and Miller developed a useful statistical approach for obtaining the $P D I$ without the need to calculate the molecular weight distributions. ${ }^{[\mathrm{S} 8]}$

At a lower temperature, the reactive points can be activated due to the increased hydrophobicity at both ends of the subunits. Here, we consider the polymerization of subunits in which the two reactive points with different activity are denoted by A and B. In the course of supramolecular polymerization, A reactive point can react stochastically with both A and B reactive points. Therefore, there are three different types of reaction in this supramolecular polymerization, which are reactions of A-to-A, A-to-B and B-to-B. ${ }^{\left[{ }^{[11]}\right.}$ The total number of subunits in the initial system is set as $N$, and the subunit mass is $M$. We introduce a parameter $\alpha$ which is the ratio of activity between A and B reactive points. The contributions of the three reaction types, A-to-A, A-to-B, and B-to-B to the extent of polymerization $p$ are $\frac{\alpha^{2}}{(\alpha+1)^{2}}, \frac{2 \alpha}{(\alpha+1)^{2}}$, and $\frac{1}{(\alpha+1)^{2}}$, respectively. The extents of polymerization for $\mathrm{A}$ and $\mathrm{B}$ reaction points were respectively written as

$$
\begin{aligned}
& p_{A}=\frac{2 \alpha}{\alpha+1} p \\
& p_{B}=\frac{2}{\alpha+1} p
\end{aligned}
$$

Noted that, these equations are valid at the initial stage of polymerization where neither reactive point A nor reactive point B is totally consumed. In other words, the following relationships should be satisfied: $0<p_{A}<1$ and $0<p_{B}<1$. Therefore, the valid range of values of $p$ can be obtained: $0<p<\frac{\alpha+1}{2 \alpha}$.

By picking the subunits (i.e. monomers) in the middle of a random polymerized nanowire (i.e. chain), we denoted the expected masses of chains which contain a randomly selected $\mathbf{A}$ or $\mathbf{B}$ ends as 
$w_{A}$ or $w_{B}$, and introduced four variables $w_{A}^{i}, w_{A}^{o}, w_{B}^{i}$, and $w_{B}^{o}$. Here $w_{A}^{i}$ represents the expected mass attached to the A reactive point via looking inward (toward the B reactive point) from this $\mathrm{A}$ reactive point and $w_{A}^{o}$ represents the expected mass attached via looking outward from the A reactive point. Similarly, $w_{B}^{i}$ and $w_{B}^{o}$ represent the expected inward and outward mass attached to the B reactive point in these subunits, respectively. The following relationships hold for this system

$$
\begin{aligned}
& w_{A}=w_{A}^{i}+w_{A}^{o} \\
& w_{B}=w_{B}^{i}+w_{B}^{o} \\
& w_{A}^{i}=M+w_{B}^{o} \\
& w_{A}^{o}=p_{A}\left(\frac{\alpha}{\alpha+1} \cdot w_{A}^{i}+\frac{1}{\alpha+1} \cdot w_{B}^{i}\right) \\
& w_{B}^{i}=M+w_{A}^{o} \\
& w_{B}^{o}=p_{B}\left(\frac{\alpha}{\alpha+1} \cdot w_{A}^{i}+\frac{1}{\alpha+1} \cdot w_{B}^{i}\right)
\end{aligned}
$$

eq. (S-9) and eq. (S-10) state the inward and outward masses attached to reactive point A. Similarly, eq. (S-11) and eq. (S-12) express the inward and outward masses of reactive point $\mathbf{B}$. Due to the hypothesis that the subunits $A$ or $B$ was randomly chosen, the possibility for $\mathrm{w}_{\mathrm{A}}$ and $\mathrm{w}_{\mathrm{B}}$ is equal to $1 / 2$. Being analogous to calculation of the weight-average molecular weight in molecular polymerization, we can solve these equations and obtain the weight-average nanowire weight

$$
M_{W}=\frac{w_{A}}{2}+\frac{w_{B}}{2}=\frac{M\left[\left(2 \alpha^{2}+2\right) p+(\alpha+1)^{2}\right]}{(\alpha+1)^{2}-4 \alpha p}
$$

The number-average nanowire weight, obtained from the extent of polymerization $p$, are given by

$$
M_{n}=\frac{N \cdot M}{(1-p) N}=\frac{M}{1-p}
$$

Combining the eq. (S-13) and eq. (S-14), we can obtain the polydispersity index as

$$
P D I=\frac{M_{W}}{M_{n}}=\frac{\left[\left(2 \alpha^{2}+2\right) p+(\alpha+1)^{2}\right](1-p)}{(\alpha+1)^{2}-4 \alpha p}
$$

Additionally, we can replace $p$ in eq. (S-15) with the equation $p=1-\frac{1}{X_{\mathrm{n}}}$ and obtain

$$
P D I=\frac{\left(3 \alpha^{2}+2 \alpha+3\right) X_{n}-2 \alpha^{2}-2}{(\alpha-1)^{2} X_{n}^{2}+4 \alpha X_{n}}
$$

Hence, the relationship between $P D I$ and $X_{\mathrm{n}}$ is associated with parameter $\alpha$. When $\alpha=1.0$, eq. 
(S-16) becomes $P D I=2-\frac{1}{X_{\mathrm{n}}}$, which is reverted to the classical formula of $P D I$ in the conventional step-growth polymerization. Noted that, these theoretical equations are only valid at the initial stage of polymerization; that is, $0<p<\frac{\alpha+1}{2 \alpha}$, and $X_{\mathrm{n}}<\frac{2 \alpha}{\alpha-1}$.

Subsequently, we used eq. (S-16) to fit the data of $P D I$ as a function of $X_{\mathrm{n}}$ in experiments and simulations (Figure 3b, Figure 5b). The eq. (S-16) fits the data well, and the parameter $\alpha$ is obtained with values of 1.56 and 1.52 for the experiments and simulations, respectively. 


\subsection{Experimental observations}

\subsubsection{Temperature-dependent polymerization of the micellar subunits}

Morphologies of aggregates obtained by temperature-dependent polymerization are examined by TEM. Figure S2 shows the typical aggregates formed by spindle-like subunits with various temperatures after annealing 85 days. Obviously, the subunits are inert and no nanowire is observed at $40{ }^{\circ} \mathrm{C}$. With decreasing temperatures, the spindles tend to connect and the nanowires become longer at lower temperatures. The TEM images also revealed that the length of the nanowires under various temperatures exhibit polydispersity.
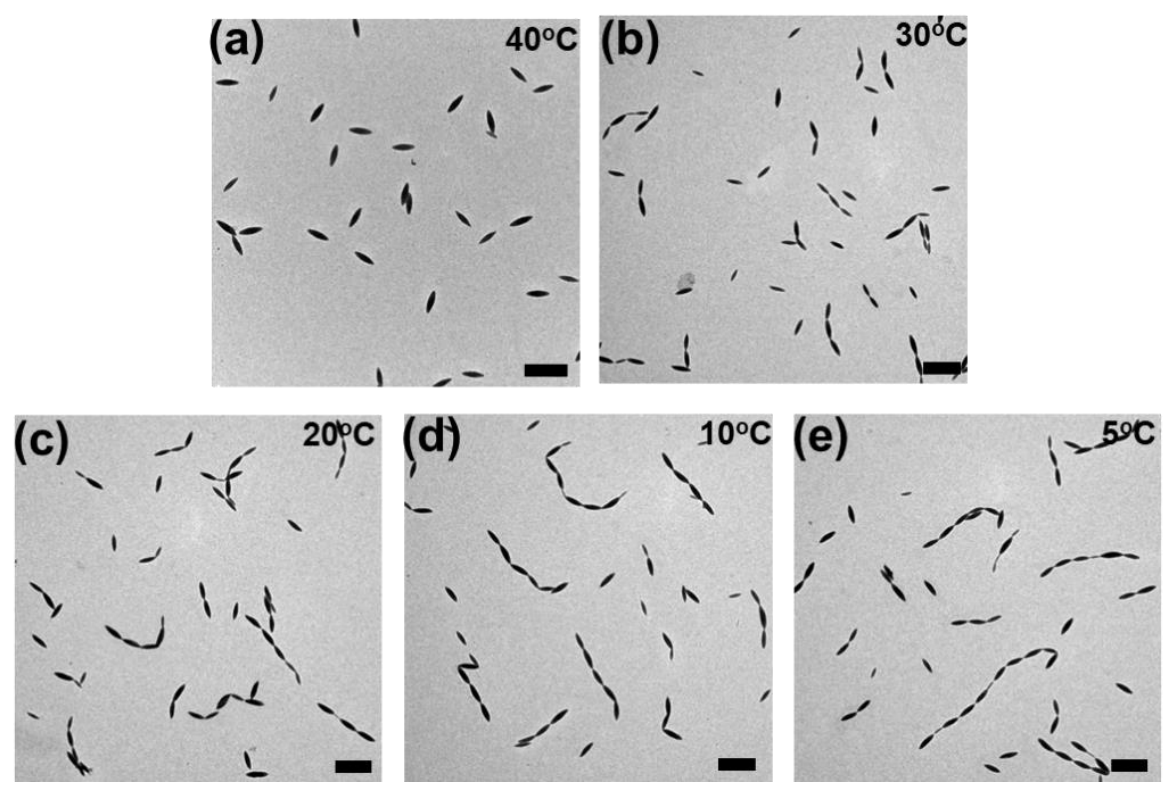

Figure S2. TEM images of the aggregates self-assembled from subunits with various self-assembly temperatures: (a) $40{ }^{\circ} \mathrm{C}$, (b) $30{ }^{\circ} \mathrm{C}$, (c) $20{ }^{\circ} \mathrm{C}$, (d) $10{ }^{\circ} \mathrm{C}$ and (e) $5{ }^{\circ} \mathrm{C}$. Scale bars: $1000 \mathrm{~nm}$.

\subsubsection{Self-assembled structures confirmed by AFM and Cryo-TEM measurements}

The nanowire morphologies were further examined by AFM. Figure S3 shows the typical morphologies of the nanowires formed by the spindle-like subunits in three dimensions. As shown in Figure S3a, the nanowires were formed through end-to-end connection of the subunits. The height profiles of the nanowires in Figure S3b and Figure S3c demonstrate that the height of the subunit 
body and the nodes between two subunits are approximately $100 \mathrm{~nm}$ and $60 \mathrm{~nm}$, indicating that the spindles were linked together. Figure S4 shows the Cryo-TEM images of the subunits (Figure S4a) and nanowires (Figure S4b) with higher resolution. The Cryo-TEM images show the same morphology as those observed by TEM, thus the drying effects on sample preparation can be ruled out.
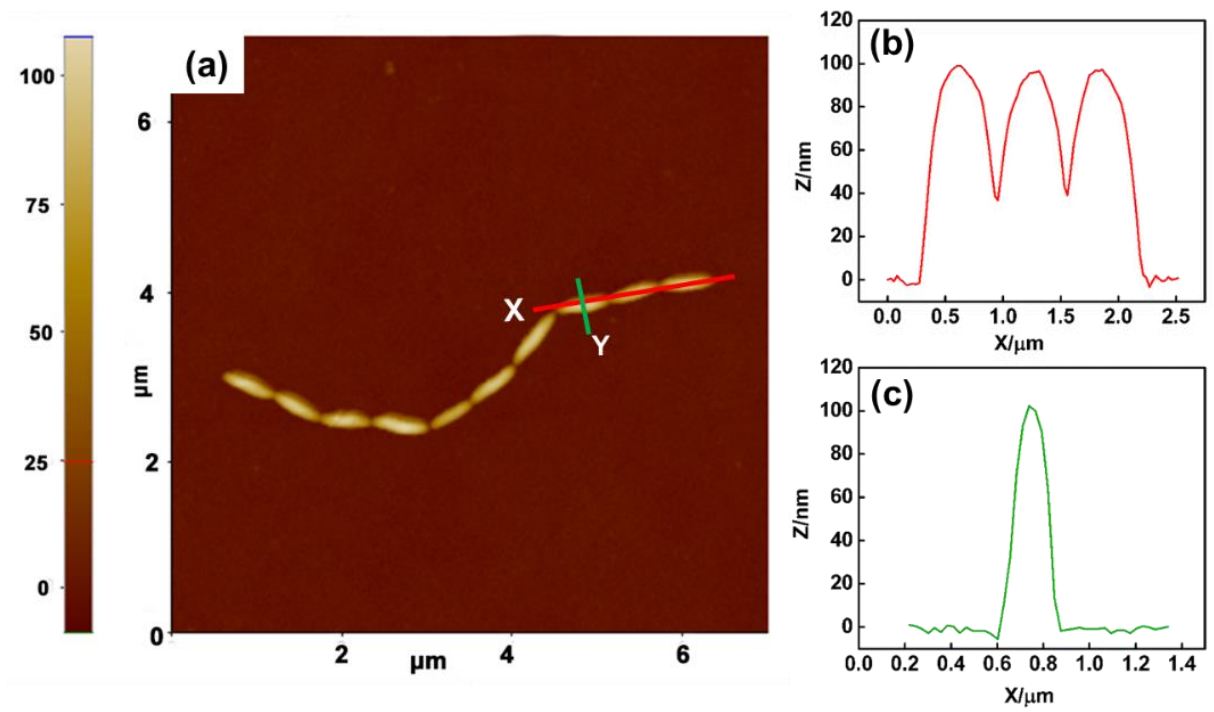

Figure S3. (a) Typical AFM image of nanowires self-assembled from the spindle-like subunits. (b) Length profile (along X direction) and (c) cross-section profile (along Y direction).
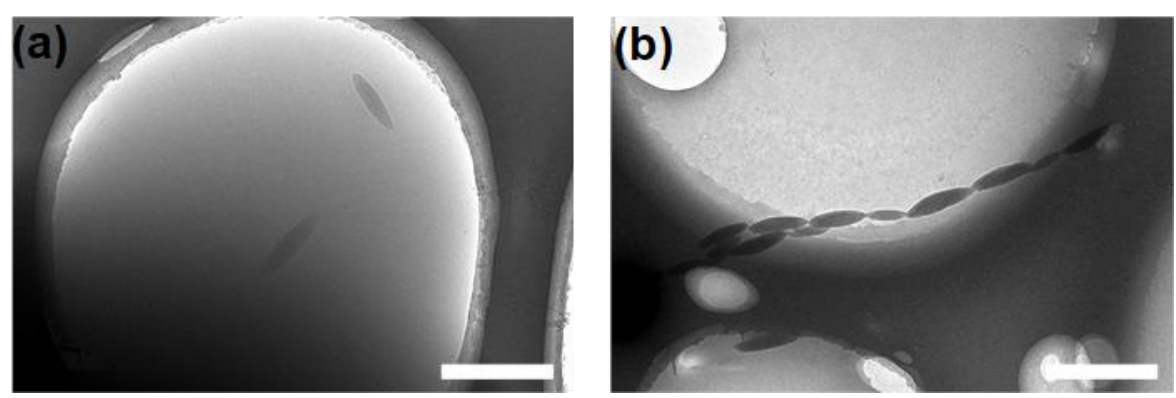

Figure S4. (a-b) Cryo-TEM images of the subunits and nanowires formed by PBLG-g-PEG-1. Scale bars: $1 \mu \mathrm{m}$. 


\subsubsection{Number fraction of the nanowires with degree of polymerization $X$}

The number fraction of the nanowires with various degree of polymerization as a function of $X$ at different temperatures is shown in Figure S5. As can be seen, the number fraction of unconnected subunits decreases and the number fraction of nanowires with more than one subunit increases with decreasing temperatures. In addition, the extent of polymerization $p$ increases as the temperatures decreases (from 0.03 to 0.76 ), indicating that more subunits joint into the supramolecular chains. It was found that $f_{\mathrm{X}}$ shows an exponential decay at each temperature (shown as the black dotted line), and the $f_{\mathrm{X}}$ data could be fit with the relationship $f_{\mathrm{X}}=(1-p) p^{X-1}$, which represents a theoretical prediction of the number fraction distribution of polymers in step-growth polymerization. Hence, the agreement between the experimental data and theoretical prediction demonstrated that the connection of subunits obey the rule of step-growth polymerization.

(a)

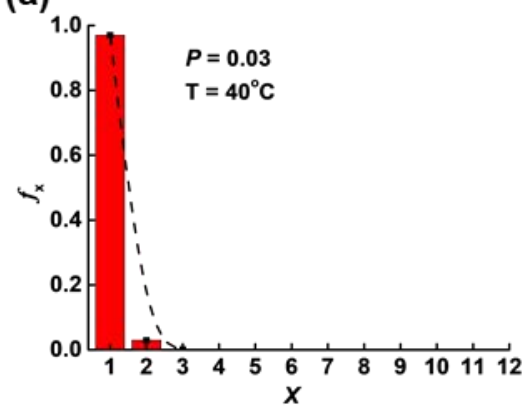

(c)

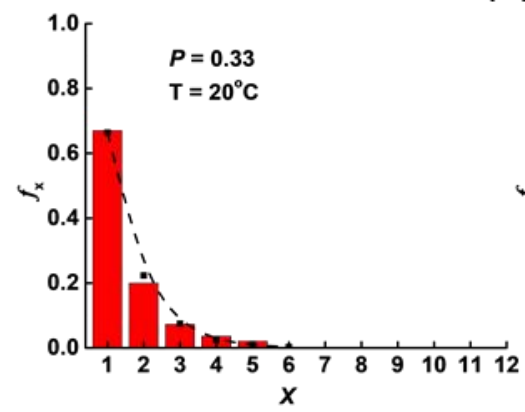

(b)

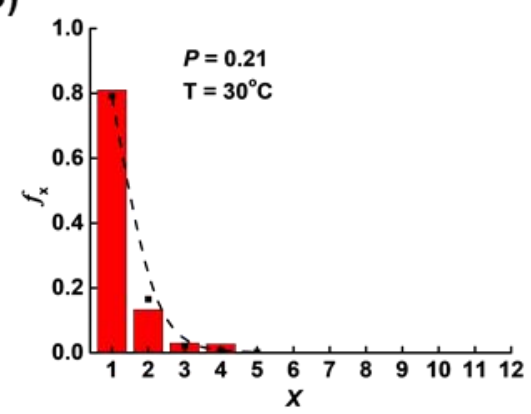

(e)
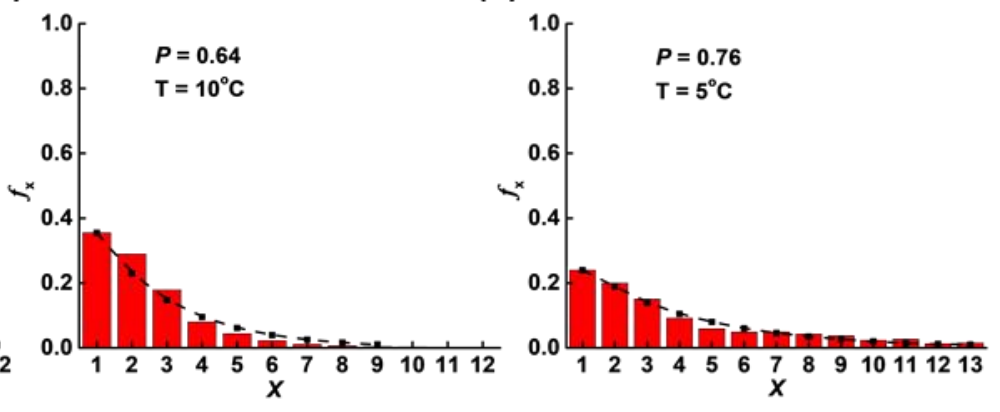

Figure S5. Number fraction, $f_{\mathrm{x}}$, of the nanowires as a function of the degree of polymerization $X$ at various temperatures after annealing for 85 days. The black dashed line is the theoretical number fraction, $f_{X}$, of the nanowires with $\mathrm{X}\left(f_{\mathrm{X}}=(1-p) p^{X-1}\right)$. 


\subsubsection{Effect of content of PBLG homopolymers on the morphologies of micellar subunits}

We have carried out an experiment to examine the effect of PBLG homopolymers on the micellar morphologies. Figures S6a-e show TEM images of the subunits assembled from the mixtures with various PBLG contents. The PBLG content varies from 0 to $75 \mathrm{wt} \%$. The statistical results of the micelle dimensions were summarized in Table S2. The molecular weights of the subunits formed with various PBLG contents were obtained by SLS measurements and also shown in Table S2 (for the details of SLS, see section 1.4.5 of the Supporting Information). These results demonstrate that the sizes and the molecular weight of the aggregates are not obviously affected by the addition of PBLG homopolymers under the present condition employed.

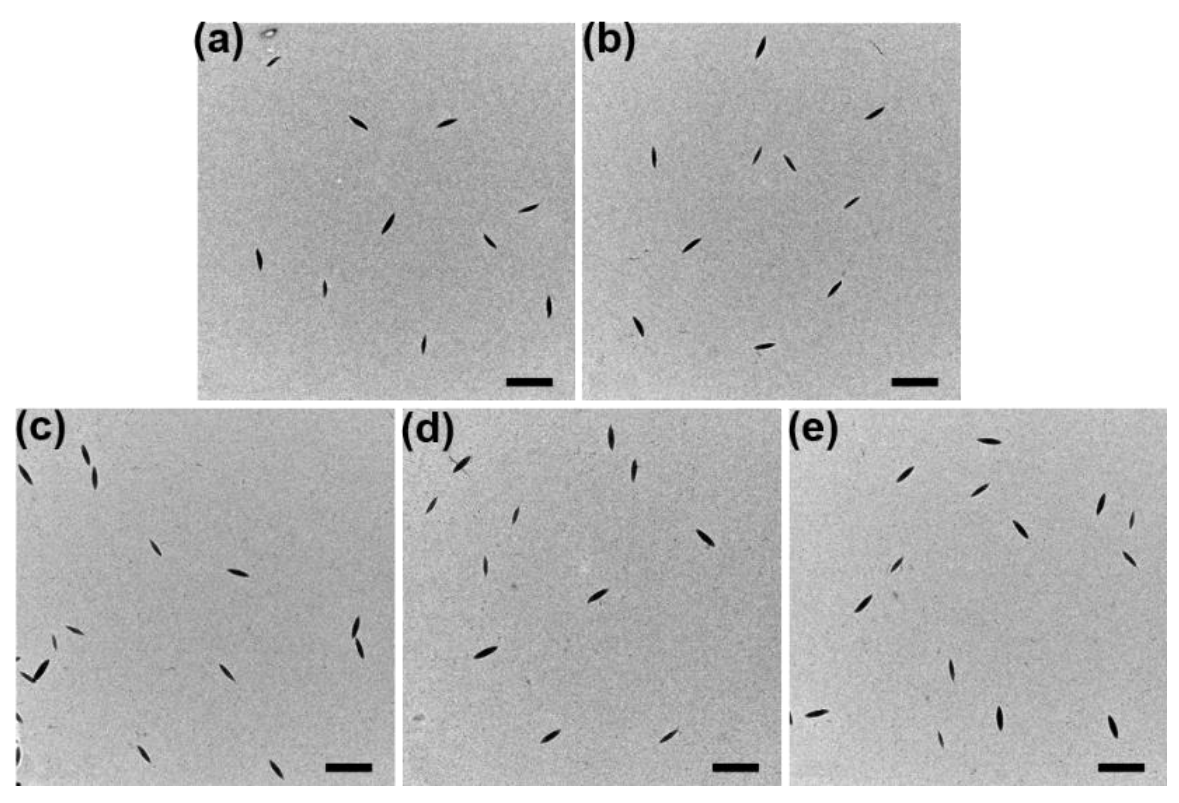

Figure S6. (a-e) TEM images of the subunits formed by PBLG-g-PEG-1 graft copolymer with various PBLG homopolymers content: (a) 0, (b) $33 \mathrm{wt} \%$, (c) $50 \mathrm{wt} \%$, (d) $67 \mathrm{wt} \%$, and (e) $75 \mathrm{wt} \%$. Scale bars: $1 \mu \mathrm{m}$. 
Table S2. Characteristics of the aggregates formed by PBLG- $g$-PEG-1 and PBLG mixture systems.

\begin{tabular}{ccccc}
\hline $\begin{array}{c}\text { PBLG-g-PEG-1 } \\
\text { and PBLG binary } \\
\text { systems }\end{array}$ & $\begin{array}{c}\text { PBLG } \\
\text { content } \\
(\mathrm{wt} \%)\end{array}$ & $\begin{array}{c}\text { Length }^{\mathrm{a}} \\
(\mathrm{nm})\end{array}$ & $\begin{array}{c}\text { Diameter } \\
(\mathrm{nm})\end{array}$ & $\begin{array}{c}M_{w}{ }^{\mathrm{sub} b} \\
(\mathrm{~g} / \mathrm{mol})\end{array}$ \\
\hline 1 & 0 & $496 \pm 26$ & $105 \pm 11$ & $2.01 \times 10^{9}$ \\
2 & 33 & $502 \pm 31$ & $112 \pm 17$ & $2.06 \times 10^{9}$ \\
3 & 50 & $498 \pm 37$ & $113 \pm 10$ & $2.11 \times 10^{9}$ \\
4 & 67 & $492 \pm 39$ & $109 \pm 12$ & $2.04 \times 10^{9}$ \\
5 & 75 & $505 \pm 24$ & $114 \pm 13$ & $2.13 \times 10^{9}$ \\
\hline
\end{tabular}

aThe length and diameter of the aggregates are obtained from at least 200 samples of TEM images. ${ }^{\mathrm{b}}$ The $M_{w}{ }^{\text {sub }}$ is obtained from SLS.

\subsubsection{Estimation of the concentration of subunits by static light scattering (SLS)}

The initial concentration of subunits in the experiments should be firstly estimated for the kinetic studies. In the $5.0 \mathrm{~mL}$ initial solution of PBLG-g-PEG-1 graft copolymers $\left(M_{w}=120130\right.$ $\mathrm{g} / \mathrm{mol}$ ), the mass concentration of polymer chains was $0.8 \mathrm{~g} / \mathrm{L}$. Therefore, the number of polymer chains in the initial solution could be obtained by

$N_{\text {polymer }}=C V_{\text {polymer }} / M_{w}=0.8 \mathrm{~g} / \mathrm{L} \times 0.005 \mathrm{~L} \div 120130 \mathrm{~g} / \mathrm{mol}=3.33 \times 10^{-8} \mathrm{~mol}$

where $C$ and $V_{\text {polymer }}$ are the initial mass concentration and solution volume, respectively.

To obtain the concentration of subunits, the average aggregation number of polymers in one subunit $\left(N_{\text {agg }}\right)$ was estimated by static light scattering (SLS) measurements. In the SLS, the angular dependence of the excess absolute time-average scattered intensity of the dilute micelle solutions was measured. In this work, the SLS light scattering experiment was carried out with a commercial LLS spectrometer (ALV/CGS-5022) equipped with an ALV-High QE APD detector and an ALV-5000 digital correlator using a He-Ne laser (the wavelength $\lambda=632.8 \mathrm{~nm}$ ) as light source. We plotted the SLS data with the form factor of an ellipsoid in the SLS characterization. The SLS measurement was performed at scattering angles from $30^{\circ}$ to $150^{\circ}$ (in increments of $2^{\circ}$ ) at $25^{\circ} \mathrm{C}$. 
The light scattering data were treated with

$K c /_{R}=\frac{1}{M P(q)}+2 A_{2} c+\cdots$

where $K, c, R, M, P(q)$ and $A_{2}$ are the optical constant, the sample concentration, the excess Rayleigh ratio, the particle molar mass, the form factor of the aggregates and the second virial coefficient, respectively. The term $q$ is the scattering wave vector with magnitude given by $q=\frac{4 \pi n_{0}}{\lambda} \sin (\theta / 2)$, where $n_{0}, \lambda$ and $\theta$ are the refractive index of the solvent, the laser wavelength and the scattering angle, respectively. ${ }^{[\mathrm{S} 12-\mathrm{S} 14]}$

When the concentration $c$ tends to be 0 , the equation (S-17) can be given by

$(R / K c)_{c \rightarrow 0}=M P(q)$

To characterize the spindles, the model of ellipsoid of revolution was used. ${ }^{[\mathrm{S} 15, \mathrm{~S} 16]}$ The form factor for the ellipsoid of revolution can be expressed as:

$P(q)=\int_{0}^{\frac{\pi}{2}} F^{2}\left[q R^{\prime}(R, \varepsilon, \alpha)\right] \sin \alpha d \alpha$

$R^{\prime}(R, \varepsilon, \alpha)=R\left(\sin ^{2} \alpha+\varepsilon^{2} \cos ^{2} \alpha\right)^{1 / 2}$

$F(u)=\frac{3[\sin (u)-u \cos (u)]}{u^{3}}, u=q R^{\prime}(R, \varepsilon, \alpha)$

where $R, \varepsilon$ and $\alpha$ are the semi axes, the axial ratio of the ellipsoid and the inclination angle of the spherical coordinates in reciprocal space.

Figure S7 shows the SLS data of the spindle-like subunits and the line is the fitting curve using Equation (S-18) according to the model of ellipsoid of revolution. As shown in Figure S7, the scattering data of the spindles can be described by the model of ellipsoid of revolution. The $R$ and $\varepsilon$ were calculated to be $51 \mathrm{~nm}$ and 5.4, respectively. Therefore, the diameter and length of the subunits obtained in SLS studies were $102 \mathrm{~nm}$ and $550 \mathrm{~nm}$, which agrees with the TEM observations $(\sim 100$ $\mathrm{nm}$ in diameter and $\sim 500 \mathrm{~nm}$ in length). 
Then the average aggregation number of PBLG- $g$-PEG chains per subunits can be obtained by

$N_{\text {agg }}=\rho V N_{A} / M_{w}$

where $\rho, V, N_{A}$, and $M_{w}$ are the density of the aggregate, the volume of the subunits, the Avogadro constant and the weight average molecular weight of graft copolymers, respectively. The $\rho$ value is $1.263 \mathrm{~g} / \mathrm{cm}^{3}$, which is calculated according to the weight fractions and the densities of the PBLG and PEG segments (the weight faction: $f_{\mathrm{PBLG}}=0.93, f_{\mathrm{PEG}}=0.03$; the densities: $\rho_{\mathrm{PBLG}}=1.278 \mathrm{~g} / \mathrm{cm}^{3}, \rho_{\mathrm{PEG}}$ $\left.=1.092 \mathrm{~g} / \mathrm{cm}^{3}\right) \cdot{ }^{[\mathrm{S} 17, \mathrm{~S} 18]}$ Therefore, the $N_{\mathrm{agg}}$ of the subunit was calculated to be $1.63 \times 10^{4}$.

As reported in the literatures, ${ }^{[\mathrm{S} 13, \mathrm{~S} 19, \mathrm{~S} 20]}$, the aggregates number per polymeric micelles can be $\sim 10$ to $\sim 10^{4}$, which is consistent with our result. The initial number of the subunits in the course of polymerization was

$N_{\text {sub }}=N_{\text {polymer }} / N_{\text {agg }}=3.33 \times 10^{-8} \mathrm{~mol} \div 1.63 \times 10^{4}=2.03 \times 10^{-12} \mathrm{~mol}$

The volume of the solution ( $\left.V_{\text {solution }}\right)$ was $5.7 \mathrm{~mL}$ for the subunits after the addition of selective solution $\left(\mathrm{H}_{2} \mathrm{O}\right)$. As a result, the molar concentration of the subunits was

$C_{0}=N_{\text {sub }} / V_{\text {solution }}=2.03 \times 10^{-12} \mathrm{~mol} \div 0.0057 \mathrm{~L}=3.56 \times 10^{-10} \mathrm{~mol} / \mathrm{L}$

Other concentrations of subunits for the kinetic study were obtained by diluting the subunits of $C_{0}$ using the same solvent for the initial micelles. 


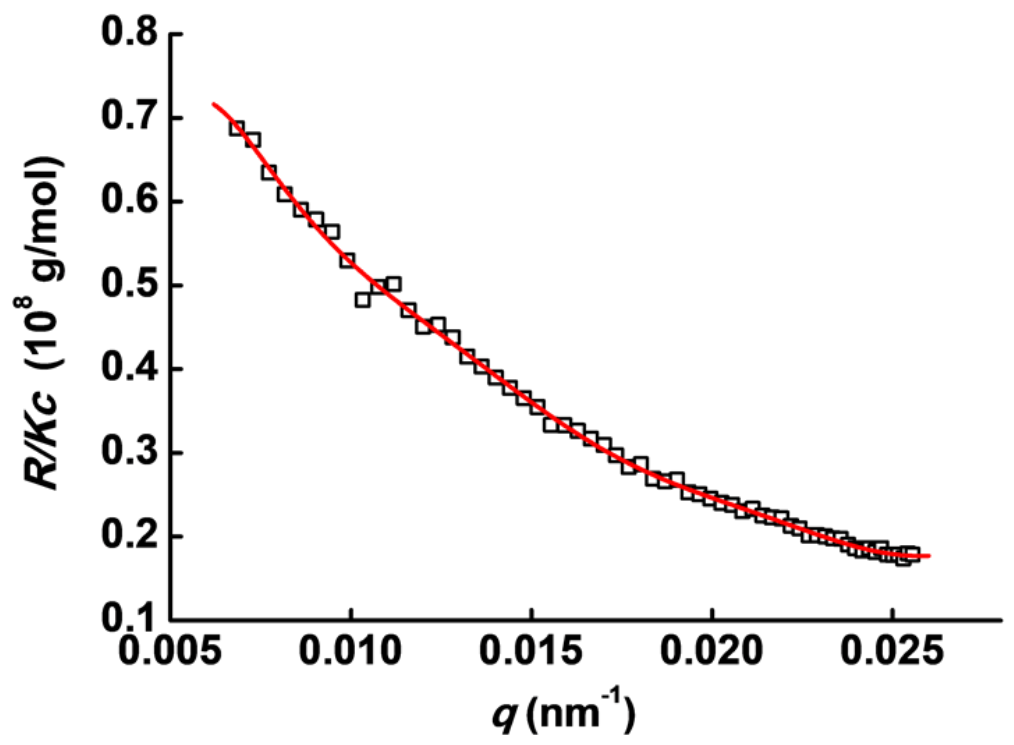

Figure S7. SLS profiles of the spindle-like subunits obtained at $25{ }^{\circ} \mathrm{C}$. The red line is the fitting curve according to the model of ellipsoid of revolution. 


\subsubsection{Effect of the degree of grafting and length of PEG on the formation of subunits and}

\section{nanowires}

We examined the self-assembly behavior of PBLG-g-PEG graft copolymers with various degrees of grafting. As shown in Figure S8a-d, with increasing the degree of grafting (the molecular weight of PEG was fixed at 750), the morphology of the subunit changes from spindle-like micelles to a mixture of spindles and spheres. This is because that the higher degree of grafting could break the parallel arrangement of the backbones and lead to the formation of spheres. ${ }^{[\mathrm{S} 21, \mathrm{~S} 22]}$

The effect of the length of PEG chains on the micellar morphologies was also examined. Shown in Figure S8e-h are the TEM images of micellar aggregates self-assembled from PBLG-g-PEG with the PEG molecular weights of 350, 750, 1000 and 1100, respectively. The degree of grafting of these copolymers is $\sim 2.1 \%$. It was found that spindle-like micelles were formed for all the samples, which indicates that the length of PEG chains in the range from 350 to 1100 has not obvious effect on the micelle morphologies. The obtained results and related analyses revealed that the spindle-like structures tend to be formed by the copolymers with the lower degree of grafting of PEG chains and longer PBLG backbones. 

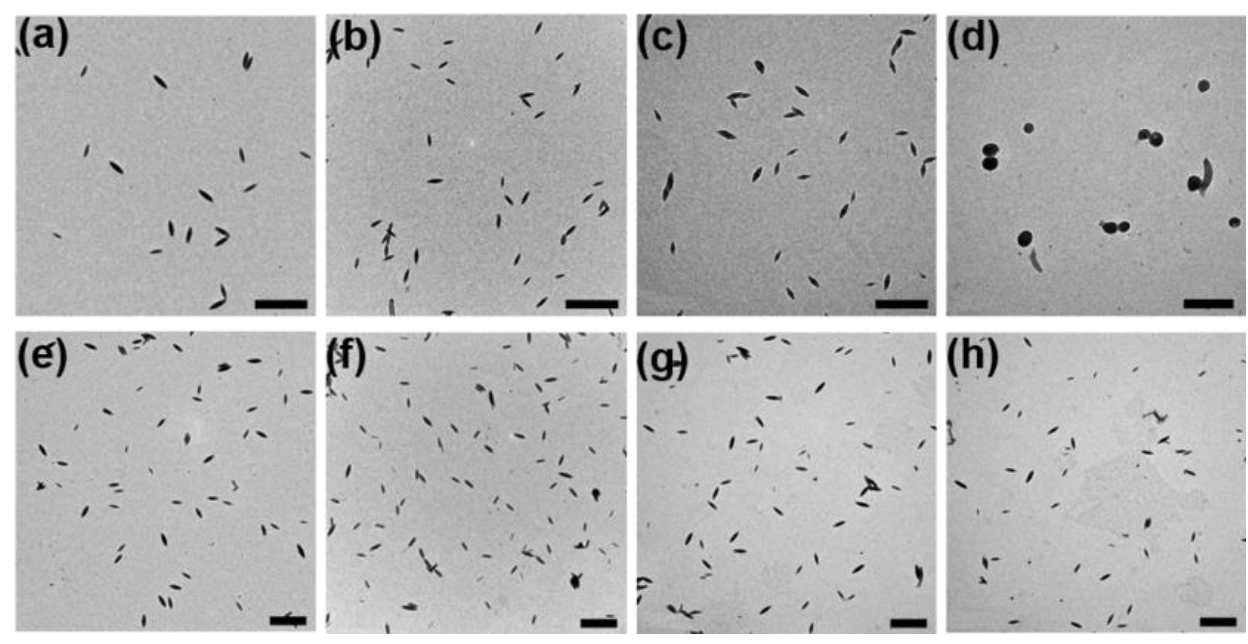

Figure S8. (a-d) TEM images of aggregates formed by PBLG-g-PEG at $40{ }^{\circ} \mathrm{C}$ with degrees of grafting of $2.33 \%, 3.08 \%, 4.24 \%$ and $8.12 \%$, respectively. The molecular weight of PEG is 750 . (e-h) TEM images of aggregates formed by PBLG-g-PEG at $40{ }^{\circ} \mathrm{C}$ with the molecular weight of PEG grafts of 350, 750, 1000 and 1100 , respectively. The degree of grafting of these copolymers is $\sim 2.1 \%$. Scale bars: $1 \mu \mathrm{m}(\mathrm{a}-\mathrm{c}, \mathrm{e}-\mathrm{h})$ and $500 \mathrm{~nm}(\mathrm{~d})$, respectively.

Then, we examined the supramolecular polymerization behavior of those subunits assembled from graft copolymers with various degrees of grafting. The subunit solution was annealed at $5^{\circ} \mathrm{C}$ for the supramolecular polymerization. As shown in Figure S9a-d, the number of subunits in the nanowires gradually decreases with increasing the degree of grafting at a fixed polymerization time of 85 days. When the degree of grafting reaches $8.12 \%$, the initial subunits become a mixture of spindles and spheres and no nanowires were formed from the subunits. Figures S9e-f show that both the number-average degree of polymerization $\left(X_{\mathrm{n}}\right)$ and the polydispersity index $(P D I)$ of the nanowires decrease with increasing the degree of grafting.

The PEG grafts form the corona of spindle micelles, while the solvophobic cores at two ends of spindles cannot be completely covered, leading to the partially exposed ends of spindles. With increasing the degree of grafting and the length of hydrophilic chain, the coverage of the PEG chains on ends of the micelles increases, which leads to the reduced reactivity. When the ends of the subunits were completely covered by the PEG chains, the reactive points at the micellar ends 
disappeared and nanowires cannot be formed (see Figure S9d). The results well support our mechanism and indicate that the reactive points at the ends of the spindles can be varied by graft contents, which is crucial for the formation of the nanowires.
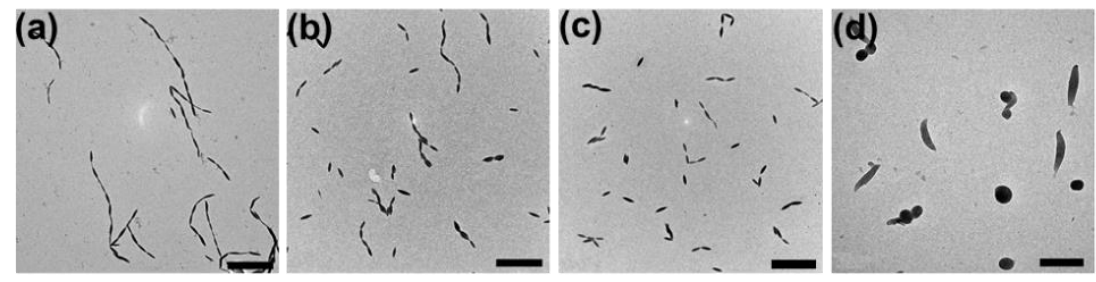

(e)

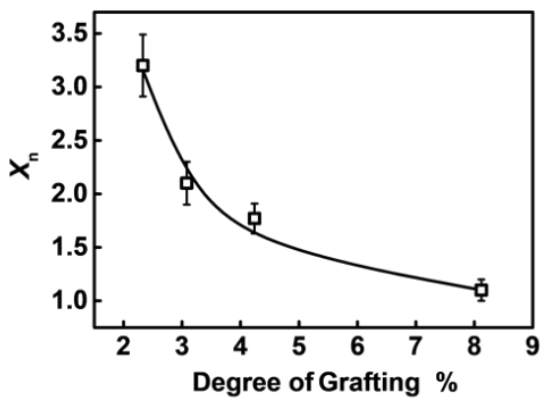

(f)

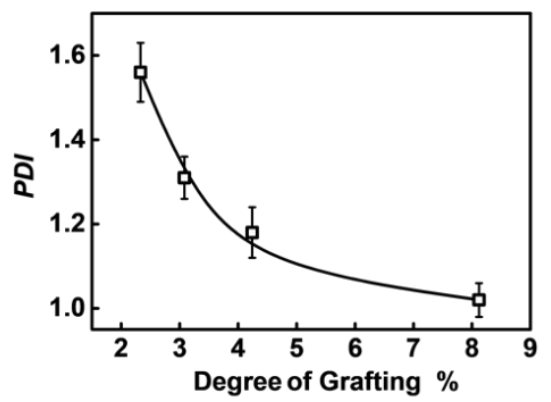

Figure S9. (a-d) TEM images of aggregates obtained by annealing the subunits with various degrees of grafting of PEG at $5{ }^{\circ} \mathrm{C}$ for 85 days: (a) $2.33 \%$, (b) $3.08 \%$, (c) $4.24 \%$ and (d) $8.12 \%$, respectively. The molecular weight of PEG is 750. (e-f) Statistics results of the number-average degree of polymerization $\left(X_{\mathrm{n}}\right)$ and the polydispersity index $(P D I)$ of the nanowires as a function of degree of grafting. Scale bars: $1 \mu \mathrm{m}(\mathrm{a}-\mathrm{c})$ and $500 \mathrm{~nm}(\mathrm{~d})$, respectively. 


\subsubsection{In situ dynamic light scattering (DLS) results of the nanowires}

To examine the growth of nanowires in solutions in situ, we carried out DLS experiment to follow the supramolecular polymerization process of the subunits formed by PBLG- $g$-PEG-1 at various temperatures. Figure S10 shows the plots of $R_{\mathrm{h}}$ versus time for the subunit solution at various temperatures. As can be seen, the $R_{\mathrm{h}}$ increases with time at those temperatures expect $40{ }^{\circ} \mathrm{C}$. And the variation rate of $R_{\mathrm{h}}$ increases as temperature decreases, which is consistent with the statistics results of the electron microscope observations (main text, Figure 3a).

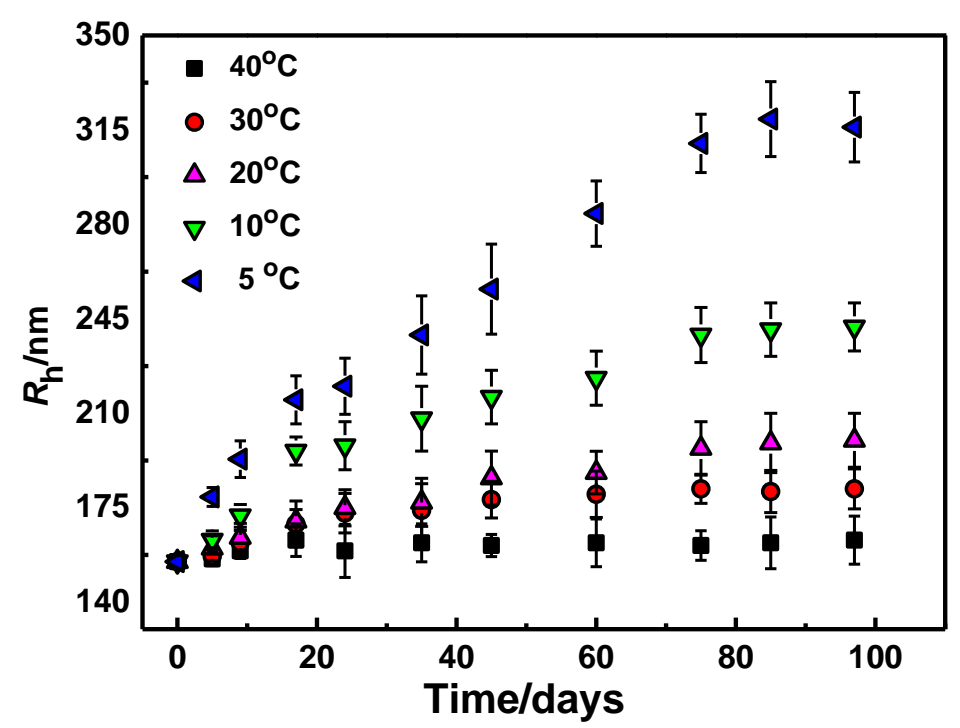

Figure S10. Plots of $R_{\mathrm{h}}$ versus time for the subunit solution at various temperature. The $R_{\mathrm{h}}$ was measured at scattering angle of $90^{\circ}$. 


\section{Theoretical simulations}

\subsection{Dissipative particle dynamics (DPD) method}

DPD is a coarse-grained particle-based mesoscopic simulation technique. It is proposed by Hoogerbrugge and Koelman in 1992. ${ }^{[\mathrm{S} 23-\mathrm{S} 25]}$ The DPD method allows for longer time and larger length scales than atomistic molecular dynamics simulations. In a DPD simulation, a block or cluster of atoms or molecules is represented by a coarse-grained DPD bead, which interacts via short-range forces. The temporal evolution of the simulated system is described via integration of Newton's equation of motion

$\frac{\mathrm{d} \mathbf{r}_{i}}{\mathrm{~d} t}=\mathbf{v}_{i}, \quad m_{i} \frac{\mathrm{d} \mathbf{v}_{i}}{\mathrm{~d} t}=\mathbf{f}_{i}$

where $\mathbf{r}_{i}, \mathbf{v}_{i}$ and $m_{i}$ denote the position, velocity and mass of the $i$-th bead. The total force $\mathbf{f}_{i}$ acting on $i$-th bead is the sum of conservative force $\mathbf{F}_{i j}^{\mathrm{C}}$, dissipative force $\mathbf{F}_{i j}^{\mathrm{D}}$ and random force $\mathbf{F}_{i j}^{\mathrm{R}}$

$\mathbf{f}_{i}=\sum_{j \neq i}\left(\mathbf{F}_{i j}^{\mathrm{C}}+\mathbf{F}_{i j}^{\mathrm{D}}+\mathbf{F}_{i j}^{\mathrm{R}}\right)$

The sum of force acts over all beads within a cutoff radius $r_{\mathrm{c}}$ beyond which the forces are neglected. The conservative force for nonbonded beads is described by a soft-repulsive interaction

$\mathbf{F}_{i j}^{\mathrm{C}}= \begin{cases}a_{i j}\left(1-r_{i j} / r_{c}\right) \hat{\mathbf{r}}_{i j}, & r_{i j} \leq r_{c} \\ 0, & r_{i j}>r_{c}\end{cases}$

where $a_{i j}$ is the repulsive interaction parameter between the $i$-th and $j$-th beads. $r_{i j}$ and $\hat{\mathbf{r}}_{i j}$ are the distance and unit vector between these two beads, respectively. The dissipative force proportional to the relative velocity $\mathbf{v}_{i j}$ is given by

$\mathbf{F}_{i j}^{\mathrm{D}}=-\gamma \omega^{D}\left(r_{i j}\right)\left(\cdot \mathbf{v}_{\boldsymbol{i j}}\right) \hat{\mathbf{r}}_{\boldsymbol{i} j}$

where the friction coefficient $\gamma$ governs the magnitude of the dissipative force and $\omega^{D}$ is a weight 
function. The random force acting as heat source to equilibrate the thermal motion of unresolved scales is defined as

$\mathbf{F}_{i j}^{\mathrm{R}}=-\sigma \omega^{R}\left(r_{i j}\right) \xi_{i j} \hat{\mathbf{r}}_{i j}$

where $\xi_{i j}$ is a randomly fluctuating variable with zero mean and unit variance, $\omega^{R}$ is a weight function, and $\sigma$ is the noise amplitude controlling the intensity of random force. The relationship between the weight functions $\omega^{D}$ and $\omega^{R}$ are selected to take the formula: $\omega^{D}=\left(\omega^{R}\right)^{2}=\left(1-r_{i j}\right)^{2}$ for $r_{i j}<r_{c}$. The combined effect of the dissipative and random force is a thermostat, leading to $\sigma=\left(2 \gamma k_{B} T\right)^{1 / 2}$.

The PBLG-g-PEG graft copolymers in the experimental systems is modeled by a coarse-grained polymer chain which is built with a rigid backbone and flexible side chains grafting on it. ${ }^{[\mathrm{S} 26, \mathrm{~S} 27]}$ All the conjoint beads in the graft copolymers are bonded by a harmonic spring force given by $\mathbf{F}_{i j}^{\mathrm{S}}=-\sum_{j} C^{\mathrm{S}}\left(r_{i j}-r_{e q}\right) \hat{\mathbf{r}}_{\boldsymbol{i}}$, where $C^{S}$ and $r_{e q}$ denote the spring constant and equilibrium bond length, respectively. To properly depict the rigidity of the backbone of the grafting copolymers, we added two additional spring forces and one angle force to the rigid blocks. The two spring forces are harmonic spring forces $\mathbf{F}_{i j}^{1}$ between the first and third beads in every three neighboring beads and $\mathbf{F}_{i j}^{2}$ between the first and end beads of the entire rigid block. The angle force constraining the bond angle to be $180^{\circ}$ is defined by $\mathbf{F}^{\theta}=-\nabla\left[k_{\theta}(\cos \theta-\cos \pi)^{2}\right]$. The head-to-tail distance of the rigid blocks is controlled at a fixed distance using the standard SHAKE routine to treat the head-tail bond. In this work, the spring constant is set as $C^{S}=100$ and angle constant is $k_{\theta}=20$. The equilibrium bond distances $r_{e q}$ for $\mathbf{F}_{i j}^{\mathrm{S}}, \quad \mathbf{F}_{i j}^{1}, \quad \mathbf{F}_{i j}^{2}$ are set to $0.7,1.4$, and 5.6, respectively. 


\subsection{Simulation model and parameter settings}

As shown in Scheme S2, the graft copolymers were modeled as $\mathbf{R}_{\mathbf{x}}-g-\left(\mathbf{C}_{\mathbf{y}}\right)_{\mathbf{z}}$, in which green beads $\mathbf{R}$ represent the PBLG rigid backbone and the red beads $\mathbf{C}$ denote the flexible PEG grafting chains. $x$ and $y$ represent the bead number in each rigid backbone and flexible grafting chain, respectively. $z$ denotes the number of grafting chains in each rigid backbone. In addition, the solvent beads $\mathrm{S}$ are also explicitly included in the simulating systems. In the simulations, we set the important parameters close to those of the experimental systems. The choice of important parameters is discussed and explained as follows.

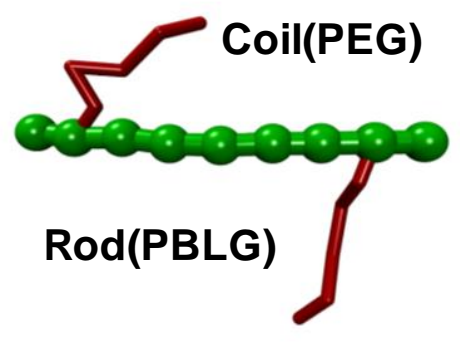

Scheme S2. DPD model of a rod-g-coil graft copolymers $\left(\mathrm{R}_{9}\left(\mathrm{C}_{5}\right)_{2}\right)$.

2.2.1 Bead number The bead number in the backbone and side chain were chosen appropriately so that their relative length matched the grafting copolymer in experiments. For the PBLG-g-PEG-1 graft copolymers used in our experimental system, the degrees of polymerization of the PBLG block and each PEG side chain were $N_{\mathrm{PBLG}}=548$ and $N_{\mathrm{PEG}}=17$, respectively. Each PBLG backbone was estimated to have 13 PEG side chains according to the degree of grafting (2.33\%). Because 3.6 BLG units form a $0.54 \mathrm{~nm}$ structure unit and $1 \mathrm{EG}$ unit occupies $0.35 \mathrm{~nm}$, the average length ratio of the rigid PBLG to the ideal PEG coil chain was $\left(N_{\mathrm{PBLG}} \times 0.54 / 3.6\right):\left(13 \times N_{\mathrm{PEG}} \times 0.35\right) \approx 1.06$. In the DPD simulations, a modeled rigid chain cannot be too long or too short. Therefore, a moderate bead number for each $\mathbf{R}$ backbone $(x=9)$ was chosen. For simplicity, the number of $\mathbf{C}$ side chains was coarse-grained to $\mathrm{z}=2$. To match the experiments, the average length ratio of the $\mathbf{R}$ backbones to the 
C side chains should satisfy the relation $(x \times 0.70):(2 \times y \times 0.70) \approx 1.06$, where 0.70 is the bond length. We can obtain a value of $y \approx 4.3$. Thus, $y$ was set as 5 in this work.

2.2.2 Interaction parameters The interaction parameters $a_{i j}$ is estimated by the relationship between the $a_{i i}$ and the Flory-Huggins parameter $\chi_{i j}$ at $\rho=3: a_{i j} \approx a_{i i}+3.497 \chi_{i j}$, where $a_{i i}=25$ denoting the interaction parameter between same species. In the first step of the self-assembly, the graft copolymers self-assemble into initial spindle-like micelles in the solvent of tetrahydrofuran (THF) $/ N, N^{\prime}$-dimethylformamide $(\mathrm{DMF}) /$ water $(5: 5: 1.4, \mathrm{v} / \mathrm{v} / \mathrm{v})$ mixture solvents at $40^{\circ} \mathrm{C}$. At this temperature, PEG is hydrophilic and compatible with the solvents, and consequently $a_{\mathrm{CS}}$ was set as 25. In contrary, PBLG is incompatibility with the solvents, and the interaction parameter between $\mathbf{R}$ block and solvent is set as $a_{\mathrm{RS}}=50$. In addition, $a_{\mathrm{RC}}$ is set to 40 , since PBLG is more compatible with PEG than the solvents.

In the simulation, the micelle subunits are assembled from rod-coil graft copolymers, where the initial states of the copolymers are disordered. For the assembled micelles, we defined the ratio of the exposed areas at both ends of the subunits as $\zeta=\mathrm{S}_{\mathrm{A}} / \mathrm{S}_{\mathrm{B}}, \mathrm{S}_{\mathrm{A}}>\mathrm{S}_{\mathrm{B}}$, where the $\mathrm{S}_{\mathrm{A}}$ and $\mathrm{S}_{\mathrm{B}}$ are the exposed areas at two subunit ends, respectively. We examined the distributions of $\zeta$ for the micelle subunits obtained in the simulations. The results are shown in Figure S11. It can be seen that most of the subunits have a value of $\zeta>1.0$ and the average value of $\zeta$ reaches to $\sim 1.5$. The different exposure areas lead to the different reactivity of the two subunit ends. And this difference in reactivity can give rise to the deviation of kinetics in the supramolecular polymerization from the theoretical model of the molecular polymerization (Figure $3 \mathrm{~b}$ in the main text). 


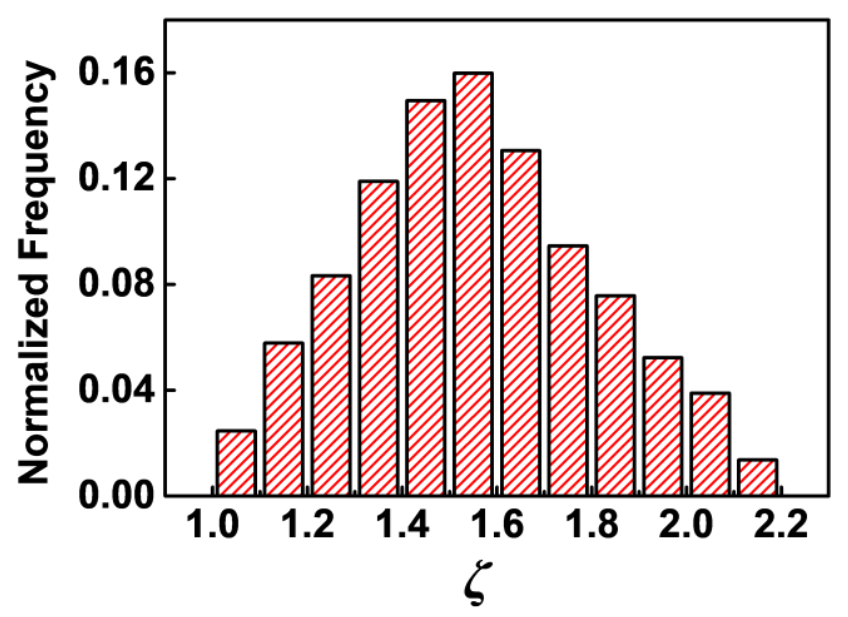

Figure S11. Distributions of the ratio $\zeta$ of the subunits obtained from the simulations.

In the subsequent assembly, to mimic the reduction of temperature to $T=30{ }^{\circ} \mathrm{C}, 20{ }^{\circ} \mathrm{C}, 10{ }^{\circ} \mathrm{C}$ and $5{ }^{\circ} \mathrm{C}$, the interaction parameters between $\mathrm{R}$ and $\mathrm{S}$ beads are respectively reset to $a_{R S}=60.0,70.0$, 80.0 and 90.0 owing to the increase of solvophobicity of PBLG backbones in solvents. The other interaction parameters remain unchanged because of their week dependence on temperature. In addition, the molecular exchange among the spindle-like subunits in the self-assembly was hindered. To simulate this behavior, we fixed the cores of the spindles obtained in the first step of the self-assembly as rigid bodies. Therefore, the molecular exchange among the spindles can be effectively prevented.

2.2.3 Computing details To guarantee the sufficient number of the subunits, all the simulations were performed with 648000 DPD beads in a cubic box $\left(60 r_{c}\right)^{3}$ under the periodic boundary conditions. A NVT ensemble was adopted. In the present simulations, all the polymer and solvent beads were of the same mass of $\mathrm{m}=1$. The interaction cutoff radius was set as $r_{\mathrm{c}}=1$. The energy scale is $k_{\mathrm{B}} T=1$, where $k_{\mathrm{B}}$ is the Boltzman constant and $T$ is the temperature. The time unit $\tau$ is defined as $\tau=$ $\left(m r_{c}^{2} / k_{B} T\right)^{1 / 2}$. The DPD step was set as $\delta t=0.04 \tau$, and the friction coefficient $\gamma$ and noise 
amplitude $\sigma$ were set as 4.5 and 3.0, respectively. In the self-assembly of grafting polymer into subunits, it was fast to obtain the equilibrium structures. Therefore, the typical DPD simulations with $10^{5}$ steps were carried out to guarantee the equilibrium of structures. The polymerization of subunits into nanowires needed a much longer time scales and consequently each simulation took $4 \times 10^{6}$ steps. Simulations with different initial random configurations were also implemented to ensure the simulations were not accidental. 


\section{References:}

[S1] Blout, E.; Karlson, R. H., Polypeptides. III. The Synthesis of High Molecular Weight Poly- $\gamma$-benzyl-L-glutamates. J. Am. Chem. Soc. 1956, 78, 941-946.

[S2] Cai, C.; Li, Y.; Lin, J.; Wang, L.; Lin, S.; Wang, X.-S.; Jiang, T., Simulation-Assisted Self-Assembly of Multicomponent Polymers into Hierarchical Assemblies with Varied Morphologies. Angew. Chem. Int. Ed. 2013, 52, 7732-7736.

[S3] Cai, C.; Lin, J.; Chen, T.; Tian, X., Aggregation Behavior of Graft Copolymer with Rigid Backbone. Langmuir 2010, 26, 2791-2797.

[S4] Cai, C.; Lin, J.; Zhu, X.; Gong, S.; Wang, X.-S.; Wang, L., Superhelices with Designed Helical Structures and Temperature-Stimulated Chirality Transitions. Macromolecules 2016, $49,15-22$

[S5] Zhuang, Z.; Jiang, T.; Lin, J.; Gao, L.; Yang, C.; Wang, L.; Cai, C., Hierarchical Nanowires Synthesized by Supramolecular Stepwise Polymerization. Angew. Chem. Int. Ed. 2016, 55, $12522-12527$.

[S6] Kratohvil, J. P., On Solution Properties of Poly( $\gamma$-benzyl-L-glutamate). Colloid Polym. Sci. 1970, $238,455-459$.

[S7] Higashihara, T.; Faust, R., Synthesis of Novel ABA Triblock and (ABA) $)_{\mathrm{n}}$ Multiblock Copolymers Comprised of Polyisobutylene and Poly( $\gamma$-benzyl-L-glutamate) Segments. React. Funct. Polym. 2009, 69, 429-434.

[S8] Macosko, C. W.; Miller, D. R., A New Derivation of Average Molecular Weights of Nonlinear Polymers. Macromolecules 1976, 9, 199-206.

[S9] Ozizmir, E.; Odian, G., Kinetics of Step Polymerization with Reactants of Unequal Functional Group Reactivity. J. Polym. Sci., A: Polym. Chem. 1980, 18, 1089-1097.

[S10] Odian, G., Principles of Polymerization. John Wiley \& Sons: 2004. 
[S11] Lu, Y.; Gao, L.; Lin, J.; Wang, L.; Zhang, L.; Cai, C., Supramolecular Step-Growth Polymerization Kinetics of Pre-Assembled Triblock Copolymer Micelles. Polym. Chem. 2019, 10, 3461-3468.

[S12] Wang, X.; Guerin, G.; Wang, H.; Wang, Y.; Manners, I.; Winnik, M. A., Cylindrical Block Copolymer Micelles and Co-Micelles of Controlled Length and Architecture. Science 2007, $317,644-647$.

[S13] Liu, G.; Yan, X.; Duncan, S., Polystyrene-block-Polyisoprene Nanofiber Fractions. 1. Preparation and Static Light-Scattering Study. Macromolecules 2002, 35, 9788-9793.

[S14] Fu, J.; Wu, C., Laser Light Scattering Study of the Degradation of Poly(sebacic anhydride) Nanoparticles. J. Polym. Sci., Part B: Polym. Phys. 2001, 39, 703-708.

[S15]Pedersen, J. S., Analysis of Small-Angle Scattering Data from Colloids and Polymer Solutions: Modeling and Least-Squares Fitting. Adv. Colloid Interface Sci. 1997, 70, 171-210.

[S16] Roig-Solvas, B.; Brooks, D.; Makowski, L., A Direct Approach to Estimate the Anisotropy of Protein Structures from Small-Angle X-Ray Scattering. J. Appl. Cryst. 2019, 52, 274-283.

[S17] Papadopoulos, P.; Floudas, G.; Schnell, I.; Lieberwirth, I.; Nguyen, T. Q.; Klok, H. A., Thermodynamic Confinement and $\alpha$-Helix Persistence Length in Poly( $\gamma$-benzyl-L-glutamate)- $b$-poly(dimethyl siloxane)- $b$-poly( $\gamma$-benzyl-L-glutamate) Triblock Copolymers. Biomacromolecules 2006, 7, 618-626.

[S18] Tang, H.; Ling, Y.; Deng, Y.; Zhang, D., Synthesis and Solid-State Self-Assembly of Poly(ethylene glycol)- $b$-poly( $\gamma$-benzyl-L-glutamate)s and Single-Walled Carbon Nanotubes. $J$. Polym. Sci., Part A: Polym. Chem. 2014, 52, 1905-1915.

[S19] Massey, J.; Power, K. N.; Manners, I.; Winnik, M. A., Self-assembly of a Novel Organometallic-Inorganic Block Copolymer in Solution and the Solid State: Nonintrusive Observation of Novel Wormlike Poly(ferrocenyldimethylsilane)-b-poly(dimethylsiloxane) 
Micelles. J. Am. Chem. Soc. 1998, 120, 9533-9540.

[S20] Tu, Y.; Wan, X.; Zhang, D.; Zhou, Q.; Wu, C., Self-Assembled Nanostructure of a Novel Coil-Rod Diblock Copolymer in Dilute Solution. J. Am. Chem. Soc. 2000, 122, 10201-10205.

[S21] Wang, C.; Li, G.; Guo, R., Multiple Morphologies from Amphiphilic Graft Copolymers Based on Chitooligosaccharides as Backbones and Polycaprolactones as Branches. Chem. Commun. 2005, 3591-3593.

[S22] Feng, C.; Li, Y.; Yang, D.; Hu, J.; Zhang, X.; Huang, X., Well-Defined Graft Copolymers: From Controlled Synthesis to Multipurpose Applications. Chem. Soc. Rev. 2011, 40, $1282-1295$.

[S23] Connors, K. A., Chemical kinetics: The Study of Reaction Rates in Solution. VCH, New York: 1990.

[S24] Hoogerbrugge, P. J.; Koelman, J. M. V. A., Simulating Microscopic Hydrodynamic Phenomena with Dissipative Particle Dynamics. Europhys. Lett. 1992, 19, 155-160.

[S25] Koelman, J. M. V. A.; Hoogerbrugge, P. J., Dynamic Simulations of Hard-Sphere Suspensions under Steady Shear. Europhys. Lett. 1993, 21, 363-368.

[S26] Lin, S.; Numasawa, N.; Nose, T.; Lin, J., Brownian Molecular Dynamics Simulation on Self-Assembly Behavior of Rod-Coil Diblock Copolymers. Macromolecules 2007, 40, 1684-1692.

[S27] Li, Y.; Jiang, T.; Lin, S.; Lin, J.; Cai, C.; Zhu, X., Hierarchical Nanostructures Self-Assembled From a Mixture System Containing Rod-Coil Block Copolymers and Rigid Homopolymers. Sci. Rep. 2015, 5, 10137. 